\title{
Effect of the Dissolving Method on the Dissolution of Dissolving Pulp Cellulose Fibers with Different Dried-States in Different $\mathrm{NaOH} /$ additives Aqueous Solutions
}

\section{Weiwei Kong}

Tianjin University of Commerce

\section{Guangrong Yu}

Tianjin University of Commerce

Jiong xing

Tianjin University of Commerce

Rui Kong

Shih Chien University Taipei Campus: Shih Chien University

Meihua Liu

Tianjin University of Commerce

Yan SHI ( $\square$ shyan@tjcu.edu.cn )

Tianjin University of Commerce https://orcid.org/0000-0002-1540-9226

\section{Research Article}

Keywords: $\mathrm{NaOH}$ /additives aqueous solution, Direct dissolution, Freezing-thaw, Dissolving pulp, Microcrystalline cellulose

Posted Date: February 10th, 2021

DOI: https://doi.org/10.21203/rs.3.rs-167392/v1

License: (c) (1) This work is licensed under a Creative Commons Attribution 4.0 International License. Read Full License 


\title{
Effect of the Dissolving Method on the Dissolution of
}

Dissolving Pulp Cellulose Fibers with Different Dried-States in Different $\mathrm{NaOH} /$ additives Aqueous Solutions

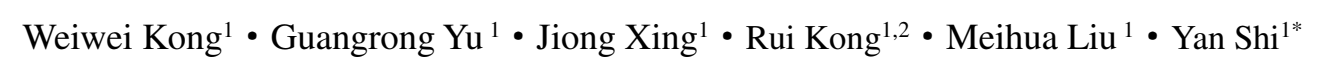

Abstract $\mathrm{A} \mathrm{NaOH/urea} \mathrm{(or} \mathrm{thiourea)} \mathrm{solvent} \mathrm{system} \mathrm{capable} \mathrm{of} \mathrm{dissolving} \mathrm{cellulose} \mathrm{at} \mathrm{lower}$ 12 temperatures is a breakthrough in cellulose chemistry, and it was reported that cellulose rapidly 13 dissolved when it was added to a precooled aqueous solution of sodium hydroxide $(\mathrm{NaOH})$ and 14 additives. Therefore, this work compared the effectiveness of the direct dissolution method and freezing-thaw method in dissolving pulp fiber and pure cellulose. Three aqueous solutions were 16 examined: $7 \% \mathrm{NaOH} / 12 \%$ urea, $9.5 \% \mathrm{NaOH} / 4.5 \%$ thiourea, and $8 \% \mathrm{NaOH} / 8 \%$ urea/6.5\% 17 thiourea. The dissolving capacity of three $\mathrm{NaOH} /$ additives aqueous solutions was analyzed 18 by polarized optical microscopy and the dissolved cellulose proportion was determined. The 19 results showed that the never-dried softwood dissolving pulp and bamboo dissolving pulp achieved better dissolution using freezing-thaw method than using direct dissolution method in the three aqueous solutions. The dissolving method had a negligible effect on the dissolution of each dissolving pulp in the $8 \% \mathrm{NaOH} / 8 \%$ urea/6.5\% thiourea solution. It seems that the direct dissolution method was more suitable for oven-dried microcrystalline cellulose with a low degree of polymerization (DP) and the freezing-thaw method was more suitable for never-dried pulp cellulose fibers with a higher DP. Keywords $\mathrm{NaOH} /$ additives aqueous solution $\cdot$ Direct dissolution $・$ Freezing-thaw $・$ Dissolving pulp • Microcrystalline cellulose

\section{Introduction} Aqueous $\mathrm{NaOH} /$ additives solutions for the dissolution of pulp cellulose have attracted wide attention from researchers because they are inexpensive, eco-friendly, and dissolve rapidly. Many researchers have reported beneficial effects from precooled $\mathrm{NaOH}$-based aqueous solutions [1-4]. The direct dissolution of cotton linter pulp cellulose in any precooled aqueous solution of $\mathrm{LiOH} / \mathrm{urea}, \mathrm{NaOH} / \mathrm{urea}$, or $\mathrm{NaOH} /$ thiourea has been found to produce a stable cellulose solution [1, 5-7]. In a study regarding the direct dissolution of cotton linter pulp cellulose in an $8 \% \mathrm{NaOH} / 8 \%$ urea/6.5\% thiourea aqueous solution precooled to $-12{ }^{\circ} \mathrm{C}$, it was found that untreated(never dried) or inactivated cellulose was able to dissolve directly and quickly [8,9]. A stable inclusion complex formed by cotton linter pulp cellulose in a $\mathrm{NaOH} /$ urea aqueous solution precooled to $-12{ }^{\circ} \mathrm{C}$ was 
$\mathrm{NaOH} / 12 \%$ urea/ $0.5 \% \mathrm{ZnO}$ aqueous solution precooled to $-13{ }^{\circ} \mathrm{C}$ has also been examined [11]. In these experiments, they investigated the dissolution of cellulose in the precooled $\mathrm{NaOH}$-based aqueous solutions using direct dissolution method, moreover, weight average molecular weight $\left(\mathrm{M}_{\mathrm{w}}\right)$ and the degree of polymerization (DP) of cellulose used are less than $1.2 \times 10^{5} \mathrm{~g} / \mathrm{mol}$ and 740 , respectively. There are also some researchers who have reported the possibility for dissolving more cellulose ( $\mathrm{DP}>1200)$ by adding additives to a precooled $\mathrm{NaOH}$ systems by direct dissolution [12] and have investigated the influence of additives on cellulose dissolution in an alkali-based solvent[13]. In addition, researchers carried out experiments about the dissolution of cellulose in $\mathrm{NaOH} /$ additives aqueous solutions using the freezing-thaw method. The solubility of cellulose from cotton linter, bagasse, alkali-soluble cellulose, and Bemcot non-woven cloth made from cotton linters in $\mathrm{NaOH} /$ urea aqueous solution using freezing-thaw method was analyzed by Zhou and Zhang [14]. The dissolution of cellulose powder $\left(\mathrm{M}_{\mathrm{w}}=1.32 \times 10^{5} \mathrm{~g} / \mathrm{mol}\right)$ in a $9 \%$ $\mathrm{NaOH} / 1 \%$ PEG aqueous solution using the freezing-thaw method has also been investigated. Namely a room temperature cellulose aqueous solution was frozen at $-15^{\circ} \mathrm{C}$ for $12 \mathrm{~h}$ and then thawed at room temperature under strong stirring, which formed a pure solution of cellulose [15]. And the solubility of wood pulp cellulose in a $10 \% \mathrm{NaOH} / 8 \%$ urea/4\% hexanolactam aqueous solution with the freezing-thaw method was found to be optimal when the mixture was frozen at $10{ }^{\circ} \mathrm{C}[16]$.

As we know, the dissolution of cellulose fibers and synthesis of cellulose derivatives on an industrial scale usually use oven-dried (OD) fibers as the starting material. Although the dissolution of OD cellulose in $\mathrm{NaOH} /$ additives using direct dissolution method is well studied, recently in our many experiments, never-dried(ND) dissolving pulp(DP $>740)$ showed better dissolution in $\mathrm{NaOH} /$ additives aqueous solution using freezing-thaw method than using direct dissolution method [17-19]. This interesting result led us to plan further experiments shown in this paper. The general thought is that fibers from the ND state will decrease the dissolving strength of the solvent because of the presence of water inside and around the fibers that dilute the aqueous solution. On the contrary, ND fibers in a swollen and more accessible state can absorb more chemicals than OD fibers. Thus, the local decrease in the solvent strength is counteracted by the opening of the structure in the ND state [20-22]. Considering that the influence of the never-dried (ND) state on the swelling and dissolution of cellulose fibers in an aqueous solution was also discussed in some detail [23]. Therefore, to understand how the ND state of a fiber affects its solubility in an aqueous solution, this work examined the dissolution of both ND and OD dissolving pulp fibers in different $\mathrm{NaOH} /$ additives aqueous solutions.

74 Materials and Methods

\section{Materials}

76 Bleached softwood sulfite dissolving pulp (SDP) was provided by Okito Kogyo Co. (Okinawa, Japan). Bleached pre-hydrolysis sulfate hardwood dissolving pulp (HDP) was provided by Hunan

78 Juntai Pulp \& Paper Co. (Huaihua, China) and bleached pre-hydrolysis sulfate bamboo dissolving samples are given in Table 1. The DP of the HDP and BDP was provided by the manufacturer, 
while the other parameters were measured in the laboratory. Microcrystalline cellulose (MCC) was purchased from Sinopharm Chemical Reagent Co. (Shanghai, China). The MCC was isolated using column chromatography (SN5318X, Sinopharm Chemical Reagent Co., Shanghai, China) and had a particle size of $20 \mu \mathrm{m}$ to $100 \mu \mathrm{m}(\mathrm{DP}<350)$. The properties of the cellulose are listed in

Table 2.

86

Table 1 Characteristics of the three commercial dissolving pulps

\begin{tabular}{|c|c|c|c|c|}
\hline \multirow{2}{*}{\multicolumn{2}{|c|}{ Parameters }} & \multicolumn{3}{|c|}{ Pulp type } \\
\hline & & SDP & HDP & $\mathrm{BDP}$ \\
\hline & Average degree of polymerization (DP) & $1520^{\mathrm{a}}$ & 800 & 1228 \\
\hline & $\alpha$-cellulose content ${ }^{\mathrm{b}}(\%)$ & 93.74 & 98.77 & 95.45 \\
\hline & Crystallinity $^{\mathrm{c}}(\%)$ & 85.0 & 86.4 & 77.2 \\
\hline Solubility in 1( & g/L $\mathrm{NaOH}$ aqueous solution $\mathrm{S}_{10}(\%)$ & 6.07 & 5.03 & 3.68 \\
\hline Solubility in 18 & $\mathrm{~g} / \mathrm{L} \mathrm{NaOH}$ aqueous solution $\mathrm{S}_{18}(\%)$ & 2.98 & 2.55 & 2.50 \\
\hline \multirow{5}{*}{$\begin{array}{l}\text { Fiber } \\
\text { quality }\end{array}$} & $0.2-7.5 \mathrm{~mm} \mathrm{~L}_{\mathrm{w}}(\mathrm{mm})$ & 1.496 & 0.587 & 1.490 \\
\hline & Fiber width $\mathrm{W}_{\mathrm{w}}(\mu \mathrm{m})$ & 37.2 & 18.2 & 21.3 \\
\hline & Content of arithmetic fine fiber(\%) & 23.2 & 39.9 & 61.6 \\
\hline & Kinks index $\left(\mathrm{mm}^{-1}\right)$ & 0.937 & 0.713 & 0.588 \\
\hline & Coarseness $(\mu \mathrm{g} / \mathrm{m})$ & 206.1 & 53.6 & 106.7 \\
\hline \multirow{5}{*}{ Neutral sugar } & Glucose content $(\%)$ & 93.03 & 97.01 & 94.62 \\
\hline & Xylose content (\%) & 1.46 & 2.04 & 1.91 \\
\hline & Mannose content (\%) & 5.51 & 0.23 & 2.40 \\
\hline & Arabinose content $(\%)$ & I & 0.72 & 0.75 \\
\hline & Galactose content (\%) & l & / & 0.32 \\
\hline
\end{tabular}

Table 2 Properties and dissolving methods of the cellulose fiber samples

\begin{tabular}{ccccc}
\hline Sample & Dissolving Method & State & Moisture $(\%)$ & $\begin{array}{c}\text { Intrinsic Viscosity } \\
(\mathrm{mL} / \mathrm{g})\end{array}$ \\
\hline SDP & Direct Dissolution & Oven-dried & 0 & 1010 \\
SDP & Direct Dissolution & Never-dried & 5.46 & 1010 \\
SDP & Freezing-thaw & Never-dried & 5.46 & 1010 \\
HDP & Direct Dissolution & Oven dried & 0 & 565 \\
HDP & Direct Dissolution & Never-dried & 6.14 & 565 \\
HDP & Freezing-thaw & Never-dried & 6.14 & 565 \\
BDP & Direct Dissolution & Oven-dried & 0 & 833 \\
BDP & Direct Dissolution & Never-dried & 6.11 & 833 \\
BDP & Freezing-thaw & Never-dried & 6.11 & 833 \\
MCC & Direct Dissolution & Oven-dried & 0 & - \\
MCC & Freezing-thaw & Oven-dried & 0 & - \\
MCC & Direct Dissolution & Never-dried & 1.48 & - \\
MCC & Freezing-thaw & Never-dried & 1.48 & - \\
\hline
\end{tabular}

*Intrinsic viscosity of each cellulose was measured based on the copper ethylenediamine method

The average DP of the SDP was measured using the copper ethylenediamine method. The $\alpha$ cellulose content was measured using TAPPI T203 cm-99. The crystallinity of the dissolving pulp was calculated using wide-angle X-ray diffraction ((D/MAX-2500, Rigaku Denki Co. Ltd., Tokyo, Japan). The solubility in $100 \mathrm{~g} / \mathrm{L}\left(S_{10}\right)$ and $180 \mathrm{~g} / \mathrm{L} \mathrm{NaOH}$ aqueous solutions $\left(S_{18}\right)$ was determined by the titrimetric method (ISO 692:1982). The $S_{10}$ value estimates the hemicellulose fraction, while the $S_{18}$ value estimates the combined hemicellulose and low- $\mathrm{M}_{\mathrm{w}}$ cellulose fraction. The fiber parameters of the three pulps were measured with a Lorentzen \& Wettre Fiber Tester 912 (Kista, 


\section{Methods}

\section{Observation of the Dissolving Behavior of the Cellulose}

138 A sample from the final pulp fibers-cellulose-solvent mixture or MCC-cellulose-solvent mixture 139 were taken using a toothpick, placed on a microscope slide, and covered with an $18-\mathrm{mm} \times 18-\mathrm{mm}$

140 glass plate. The sample was analyzed via polarized optical microscopy in the transmission mode

141 (BM-57XCC, Shanghai Biem, Shanghai, China). The magnification of the eyepiece was adjusted 142 to $4(4 \times)$.

\section{Separation of the Cellulose Solution and Insoluble Fractions}

The pulp fibers-cellulose-solvent mixture or MCC-cellulose-solvent mixture was centrifuged at $5000 \mathrm{rpm}$ for $10 \mathrm{~min}$ at $5{ }^{\circ} \mathrm{C}$ (L535-1 low-speed, Changsha Xiangyi Centrifuge Instrument Co., Changsha, China). Part of the supernatant liquid was moved to a transparent plastic sample bottle with a Pasteur pipette for the viscosity measurement. The remaining insoluble fractions in the centrifuge tube were filtrated by a glass sand core funnel and SHZ-D (III) vacuum pump (Lanphan, Henan, China). The filtered material was then washed with the corresponding $\mathrm{NaOH} /$ additives aqueous solution three times and further washed with distilled water until it was neutral.

\section{Viscosity Measurement of the Cellulose Solution}

The viscosity of cellulose solution produced by dissolution of three dissolving pulps in the different $\mathrm{NaOH} / \mathrm{additives}$ aqueous solutions was measured by a $0.9-\mathrm{mm}$ to $1-\mathrm{mm}$ Ubbelohde viscometer (Taizhou Jiaojiang District Glass Instrument Factory, Zhejiang, China). Two measurement times were recorded with a timer. One measurement was the time $(t)$ passing through two calibrated lines in the measuring bulb for the cellulose solution. The other measurement was the time $\left(t_{0}\right)$ passing through two calibrated lines in the measuring bulb for the pure $\mathrm{NaOH} /$ additives aqueous solvent at the same temperature. The ratio of $t$ to $t_{\mathrm{o}}$ was defined as $\eta_{\mathrm{r}}$. The viscosity of each cellulose solution was measured at least three times and the average was calculated. The intrinsic viscosity $([\eta])$ of the three commercial dissolving pulp samples was measured using the one-point method [5]. The kinetic energy correction was always negligible, so the $[\eta]$ value was calculated with Eq. 1 ,

$$
[\eta]=\left[2\left(\eta_{r}-1-\ln \eta_{r}\right)\right] 1 / 2 / c
$$

166 Where $\eta_{\mathrm{r}}$ is the relative viscosity, and $\mathrm{c}$ is the concentration of polymer, $\mathrm{g} / \mathrm{ml}$.

\section{Measurement of the Dissolved Proportion of the Three Dissolving Pulps}

The insoluble fractions were collected in a plastic culture dish and dried in a vacuum oven (Shanghai Senxin Experimental Instruments Co., Ltd., Shanghai, China) with anhydrous calcium chloride at $50{ }^{\circ} \mathrm{C}$ for $6 \mathrm{~h}$. The samples were then weighed and named, depending on the weight of the insoluble fractions and original pulp. The dissolved proportions of the dissolving pulp were calculated with Eq. 2,

$$
\text { Dissolved proportion }=\left(1-W_{i} / W_{o}\right) \times 100 \%
$$



and the average value was calculated.

\section{Results and Discussion} Solutions

The swelling and dissolution of the three dissolving pulp samples in different aqueous solutions were observed by polarized optical microscopy to compare their dissolving behavior. The images clearly showed that the $8 \% \mathrm{NaOH} / 8 \%$ urea/6.5\% thiourea solution had a better dissolving capacity than the $7 \% \mathrm{NaOH} / 12 \%$ urea and $9.5 \% \mathrm{NaOH} / 4.5 \%$ thiourea solutions for each dissolving pulp with a DP higher than 740 , regardless of which dissolving method was used.
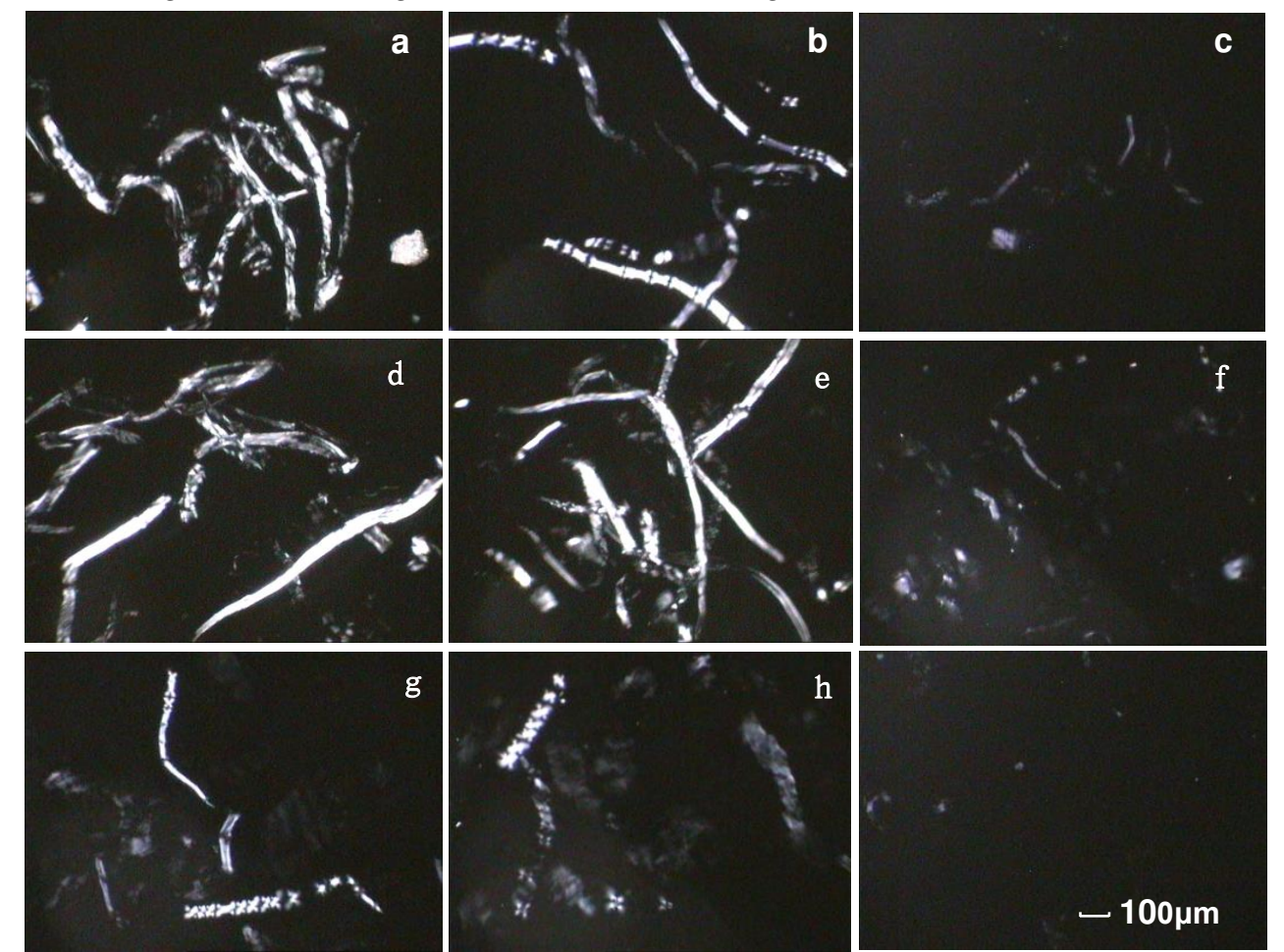

Fig.1 Polarized optical microscopy images of the SDP dissolved in the different $\mathrm{NaOH} /$ additives aqueous solutions using direct dissolution method,OD: (a) 7\% NaOH/12\% urea, (b) $9.5 \% \mathrm{NaOH} / 4.5 \%$ thiourea, and (c) $8 \% \mathrm{NaOH} / 8 \%$ urea/6.5\% thiourea; using direct dissolution method,ND: (d) $7 \% \mathrm{NaOH} / 12 \%$ urea, (e) $9.5 \%$ $\mathrm{NaOH} / 4.5 \%$ thiourea, and (f) $8 \% \mathrm{NaOH} / 8 \%$ urea/6.5\% thiourea and using freezing-thaw method,ND: (g) $7 \%$ $\mathrm{NaOH} / 12 \%$ urea, (h) $9.5 \% \mathrm{NaOH} / 4.5 \%$ thiourea, and (i) $8 \% \mathrm{NaOH} / 8 \%$ urea/6.5\% thiourea

The SDP (Fig.1) exhibited good swelling of the fibers. Using freezing-thaw method performed better than using direct dissolution method for the dissolution of SDP in each solvent. The fibers of the HDP (Fig.2) remained thin and short after treatment. There was little difference in the dissolution of the HDP between the freezing-thaw and direct dissolution methods for each solvent. The BDP fibers appeared to be long and stiff (Fig.3). The freezing-thaw method was more effective than the direct dissolution method for the dissolution of the BDP in each solvent. The dissolution of small BDP fibers left many micro-sized fibers in the solvent, which indicated that it was the most difficult to dissolve the BDP cellulose in the $\mathrm{NaOH} /$ additives aqueous solutions. These findings were consistent with the results reported by Spinu et al [23]. Namely, the ND state was more reactive for the dissolution of SDP or BDP in $\mathrm{NaOH} /$ additives aqueous solutions. The 
ND state had no remarkable effect on the HDP, which illustrated that the dissolving capacity of a

$220 \mathrm{NaOH} /$ additives aqueous solution depends on the origin of the cellulose fibers, composition of the 221 aqueous solution, and dissolving method. As mentioned in previous studies, ND cellulose fibers 222 are in a swollen and more accessible state, which means that they can absorb more chemicals than 223 OD fibers. The local decrease in the solvent strength is counteracted by the opening of the 224 structure in the ND state at different degrees, which was also affected by different $\mathrm{NaOH} /$ additives 225 aqueous solutions.
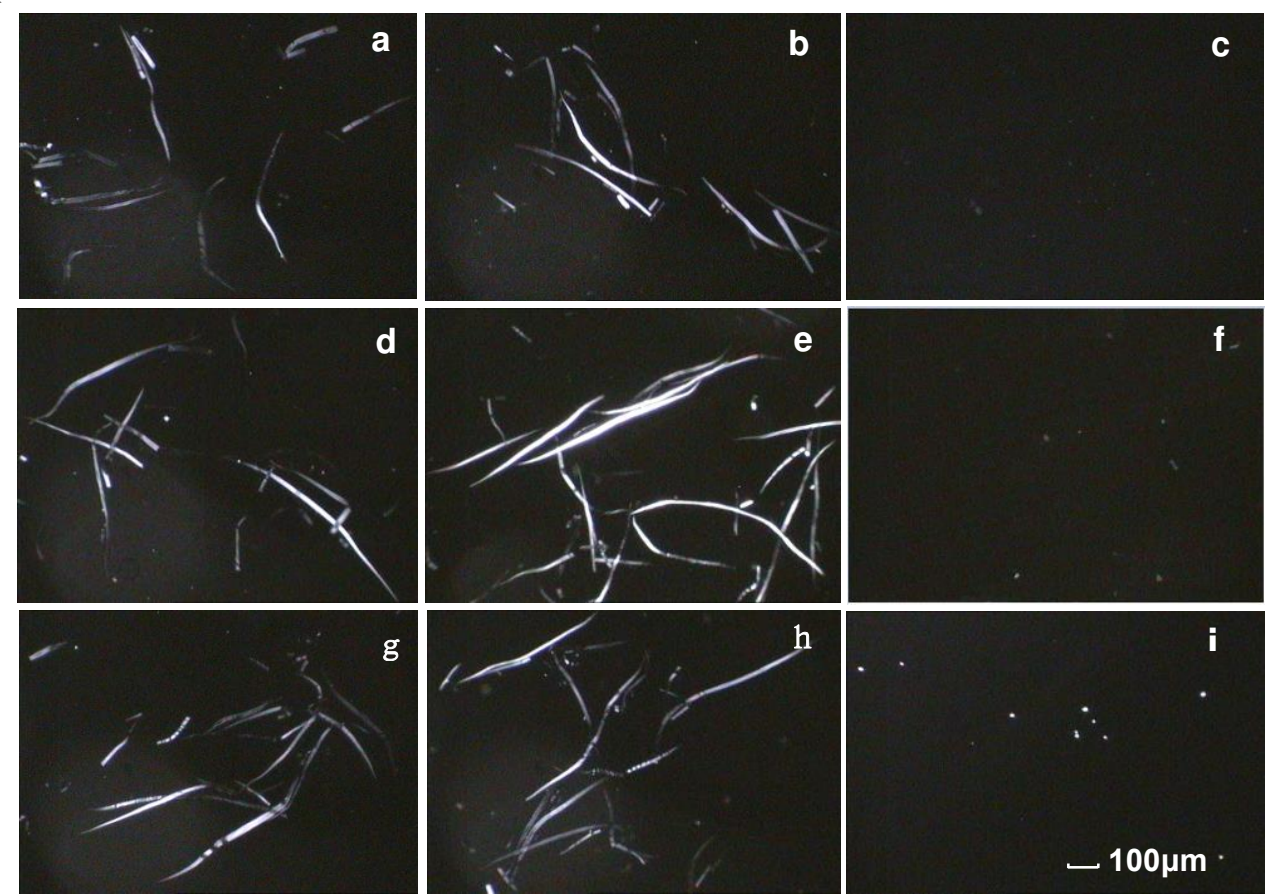

Fig.2 Polarized optical microscopy images of the HDP dissolved in the different $\mathrm{NaOH}$ /additives aqueous solutions using direct dissolution method,OD: (a) $7 \% \mathrm{NaOH} / 12 \%$ urea, (b) $9.5 \%$ $\mathrm{NaOH} / 4.5 \%$ thiourea, and (c) $8 \% \mathrm{NaOH} / 8 \%$ urea/6.5\% thiourea; using direct dissolution method,ND: (d) $7 \% \mathrm{NaOH} / 12 \%$ urea, (e) $9.5 \% \mathrm{NaOH} / 4.5 \%$ thiourea, and (f) $8 \% \mathrm{NaOH} / 8 \%$ urea/6.5\% thiourea and using freezing-thaw method,ND: (g) $7 \% \mathrm{NaOH} / 12 \%$ urea, (h) $9.5 \%$ $\mathrm{NaOH} / 4.5 \%$ thiourea, and (i) $8 \% \mathrm{NaOH} / 8 \%$ urea/6.5\% thiourea
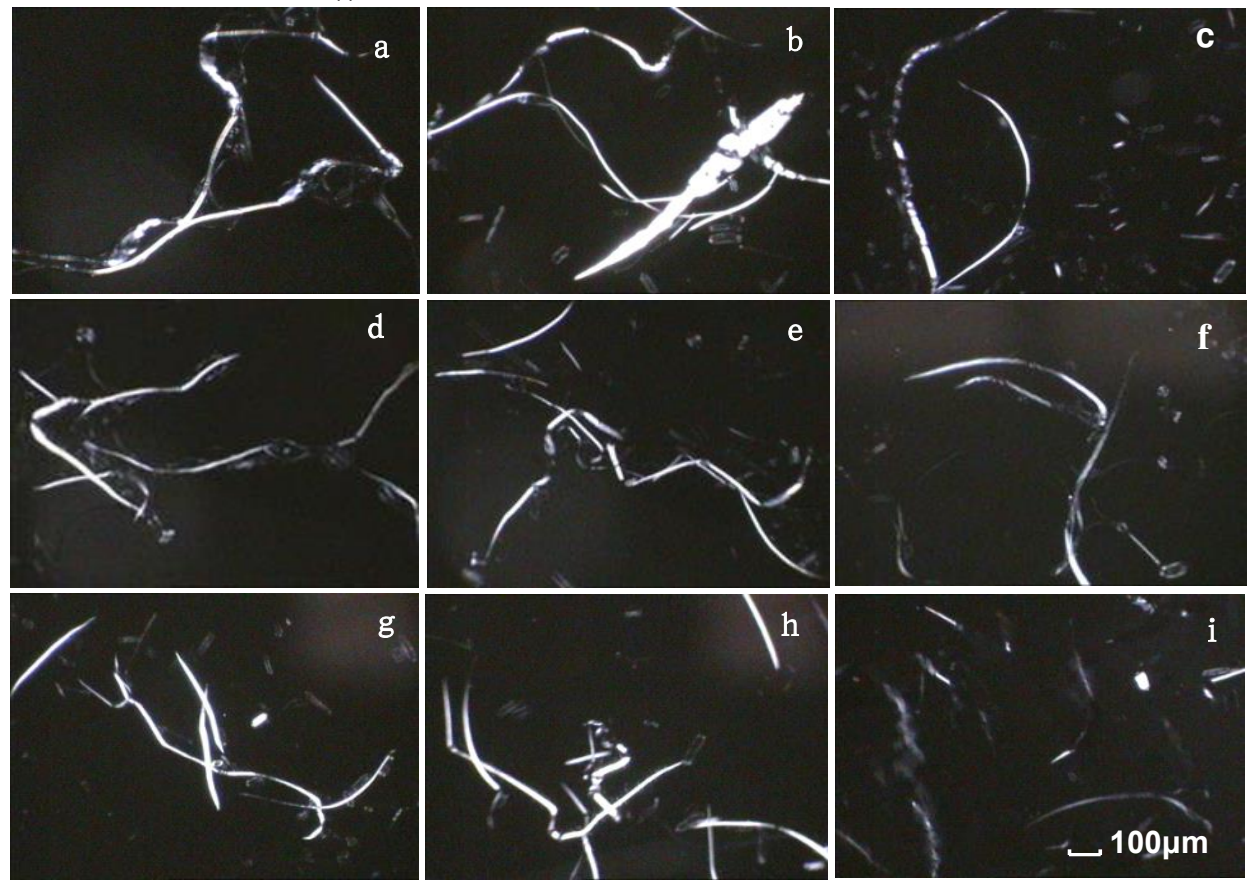

$-100 \mu \mathrm{m}$ 
Fig.3 Polarized optical microscopy images of the BDP dissolved in the different $\mathrm{NaOH} /$ additives aqueous solutions using direct dissolution method,OD: (a) $7 \% \mathrm{NaOH} / 12 \%$ urea, (b) $9.5 \% \mathrm{NaOH} / 4.5 \%$ thiourea, and (c) $8 \% \mathrm{NaOH} / 8 \%$ urea/6.5\% thiourea; using direct dissolution method,ND: (d) $7 \% \mathrm{NaOH} / 12 \%$ urea, (e) $9.5 \%$ $\mathrm{NaOH} / 4.5 \%$ thiourea, and (f) $8 \% \mathrm{NaOH} / 8 \%$ urea/6.5\% thiourea and using freezing-thaw method,ND: (g) $7 \%$ $\mathrm{NaOH} / 12 \%$ urea, (h) $9.5 \% \mathrm{NaOH} / 4.5 \%$ thiourea, and (i) $8 \% \mathrm{NaOH} / 8 \%$ urea/6.5\% thiourea

\section{Dissolving Capacity of the $\mathrm{NaOH} /$ additives Aqueous Solutions}

As we know, using polarized optical microscopy to follow the dissolution might be inaccurate because not only crystalline cellulose but a lot of non-dissolved anisotropic cellulose parts can be seen in polarized light. Thus the dissolution degree of the three dissolving pulps was evaluated by recovering the insoluble fiber fractions. The dissolved proportions of all of the samples are shown in Table 3. The dissolved proportions of the samples in the $8 \% \mathrm{NaOH} / 8 \%$ urea/6.5\% thiourea solution were all similar, regardless of which dissolving method was used. However, the dissolved proportions of the samples in the $7 \% \mathrm{NaOH} / 12 \%$ urea and $9.5 \% \mathrm{NaOH} / 4.5 \%$ thiourea solutions were higher when the freezing-thaw method was used, rather than the direct dissolution method. While there was a large difference in the solubility for these two systems, there was little difference in the solubility for the $8 \% \mathrm{NaOH} / 8 \%$ urea/6.5\% thiourea solution between the two dissolving methods.

Table 3 Dissolved proportion of each dissolving pulp in the $\mathrm{NaOH} /$ additives aqueous solutions

\begin{tabular}{llrcc}
\hline \multirow{2}{*}{ Dissolved Proportion (\%) } & \multicolumn{3}{c}{$\mathrm{NaOH} /$ additives Aqueous Solution } \\
\cline { 3 - 5 } & \multicolumn{2}{c}{$\begin{array}{l}7 \% \mathrm{NaOH} / 12 \% \\
\text { urea }\end{array}$} & $\begin{array}{l}9.5 \% \mathrm{NaOH} / 4.5 \% \\
\text { thiourea }\end{array}$ & $\begin{array}{l}8 \% \mathrm{NaOH} / 8 \% \\
\text { urea/6.5\% thiourea }\end{array}$ \\
\hline \multirow{3}{*}{ SDP } & Direct Dissolution,OD & $47.3 \pm 0.4$ & $44.4 \pm 0.5$ & $79.8 \pm 0.4$ \\
& Direct Dissolution,ND & $46.2 \pm 0.5$ & $42.1 \pm 0.3$ & $78.4 \pm 0.3$ \\
& Freezing-thaw,ND & $52.1 \pm 0.5$ & $50.5 \pm 0.4$ & $80.0 \pm 0.3$ \\
& Direct Dissolution,OD & $44.2 \pm 0.4$ & $41.7 \pm 0.4$ & $73.2 \pm 0.3$ \\
$\mathrm{HDP}$ & Direct Dissolution,ND & $43.0 \pm 0.4$ & $39.7 \pm 0.5$ & $72.0 \pm 0.4$ \\
& Freezing-thaw,ND & $43.8 \pm 0.3$ & $40.1 \pm 0.3$ & $72.2 \pm 0.4$ \\
\multirow{3}{*}{ BDP } & Direct Dissolution,OD & $43.4 \pm 0.2$ & $40.2 \pm 0.3$ & $59.4 \pm 0.2$ \\
& Direct Dissolution,ND & $41.5 \pm 0.5$ & $38.1 \pm 0.5$ & $59.0 \pm 0.3$ \\
& Freezing-thaw,ND & $45.9 \pm 0.2$ & $44.2 \pm 0.3$ & $61.4 \pm 0.4$ \\
\hline
\end{tabular}

Table 4 Viscosity of the cellulose solution from the dissolving pulp samples

\begin{tabular}{lllll}
\hline \multirow{2}{*}{\lceil]$\left(\mathrm{ml} / \mathrm{g}, 25{ }^{\circ} \mathrm{C}\right)$} & \multicolumn{3}{c}{$\mathrm{NaOH} /$ additives Aqueous Solution } \\
\cline { 3 - 5 } & & $\begin{array}{l}7 \% \mathrm{NaOH} / 12 \% \\
\text { urea }\end{array}$ & $\begin{array}{l}9.5 \% \mathrm{NaOH} / 4.5 \% \\
\text { thiourea }\end{array}$ & $\begin{array}{l}8 \% \mathrm{NaOH} / 8 \% \\
\text { urea/6.5\% thiourea }\end{array}$ \\
\hline \multirow{2}{*}{$\mathrm{SDP}$} & Direct Dissolution,OD & $330.2 \pm 1.6$ & $343.3 \pm 2.3$ & $470.6 \pm 1.7$ \\
& Direct Dissolution,ND & $310.0 \pm 2.2$ & $319.1 \pm 2.8$ & $440.5 \pm 2.7$ \\
& Freezing-thaw,ND & $340.8 \pm 2.3$ & $350.7 \pm 2.7$ & $472.2 \pm 2.5$ \\
\multirow{3}{*}{$\mathrm{HDP}$} & Direct Dissolution,OD & $310.3 \pm 2.3$ & $257.0 \pm 1.9$ & $315.1 \pm 2.2$ \\
& Direct Dissolution,ND & $300.2 \pm 0.5$ & $230.8 \pm 2.7$ & $299.7 \pm 2.0$ \\
& Freezing-thaw,ND & $304.3 \pm 2.1$ & $246.0 \pm 2.4$ & $310.4 \pm 2.7$ \\
\multirow{3}{*}{$\mathrm{BDP}$} & Direct Dissolution,OD & $120.5 \pm 0.6$ & $130.1 \pm 1.1$ & $206.1 \pm 1.4$ \\
& Direct Dissolution,ND & $120.0 \pm 1.6$ & $126.9 \pm 2.0$ & $180.2 \pm 2.2$ \\
& Freezing-thaw,ND & $149.2 \pm 2.1$ & $143.7 \pm 2.4$ & $210.0 \pm 1.8$ \\
\hline
\end{tabular}

Table 4 shows that the $8 \% \mathrm{NaOH} / 8 \%$ urea/6.5\% thiourea solution had a stronger dissolving capacity than the other two solvents for each dissolving pulp sample. The intrinsic viscosity of the cellulose solution from the $8 \% \mathrm{NaOH} / 8 \%$ urea/6.5\% thiourea solution was not too high, which indicated that the cellulose fibers in that solution partially degraded. Measurements from the dissolved proportions and the cellulose viscosity supported the polarized optical microscopy 


\section{Dissolution of $\mathrm{MCC}$ in the $\mathrm{NaOH} /$ additives Aqueous Solutions}

As a candidate of pure cellulose, the dissolution of $\operatorname{MCC}(\mathrm{DP}<350)$ in the above mentioned $\mathrm{NaOH} /$ additives aqueous solutions was also investigated. Doing this helped to further check the effect of cellulose DP on the dissolving method in the $\mathrm{NaOH} /$ additives aqueous solutions. The MCC was dried in an oven at $60{ }^{\circ} \mathrm{C}$ before being used to remove the remaining water. The cellulose sample used had a low DP to ensure a good solubility and avoid complications with noncellulose components, such as hemicellulose and lignin [24].

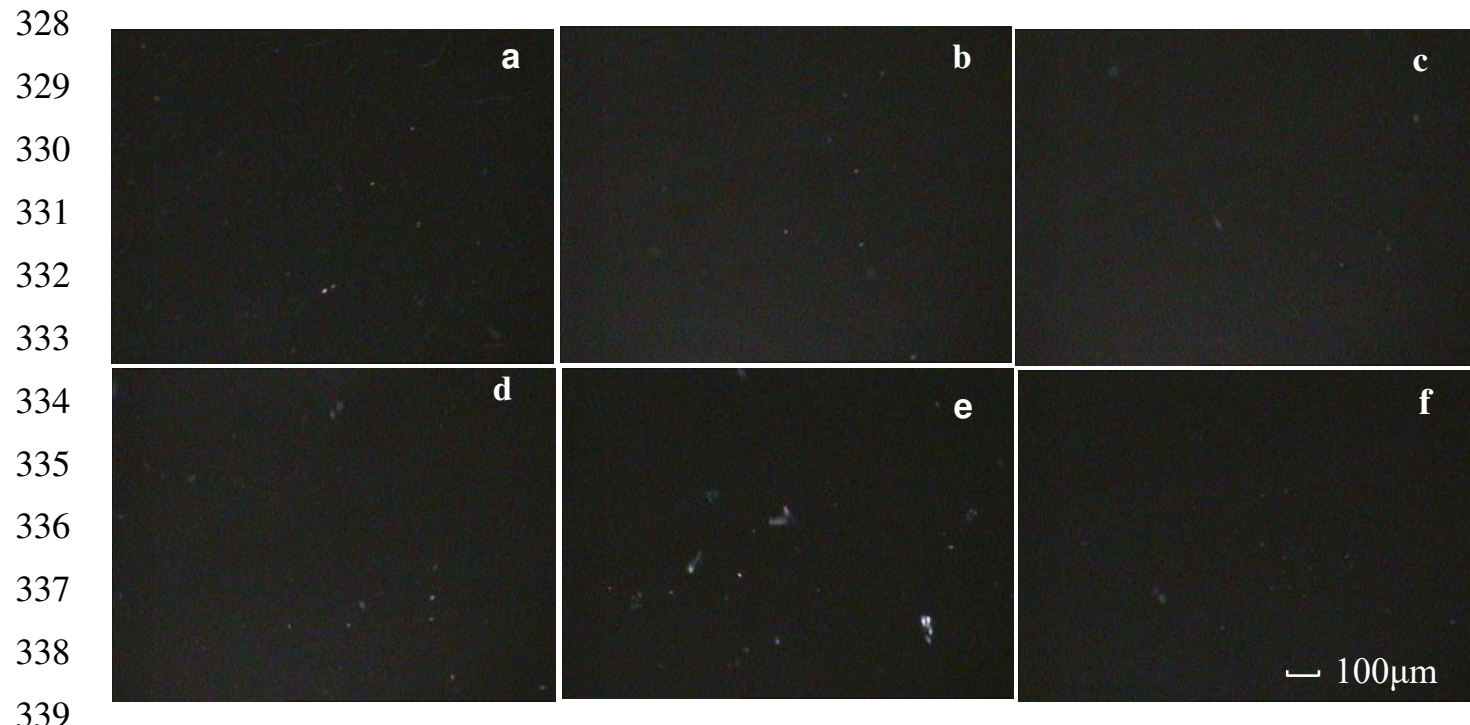

Fig.4 Polarized optical microscopy images of the OD MCC dissolved in the different $\mathrm{NaOH} /$ additives aqueous solutions with the direct dissolution method: (a) $7 \% \mathrm{NaOH} / 12 \%$ urea, (b) $9.5 \% \mathrm{NaOH} / 4.5 \%$ thiourea, and (c) $8 \% \mathrm{NaOH} / 8 \%$ urea/6.5\% thiourea; and with the freezing-thaw method: (d) $7 \% \mathrm{NaOH} / 12 \%$ urea, (e) $9.5 \% \mathrm{NaOH} / 4.5 \%$ thiourea, and (f) $8 \% \mathrm{NaOH} / 8 \%$ urea/6.5\% thiourea
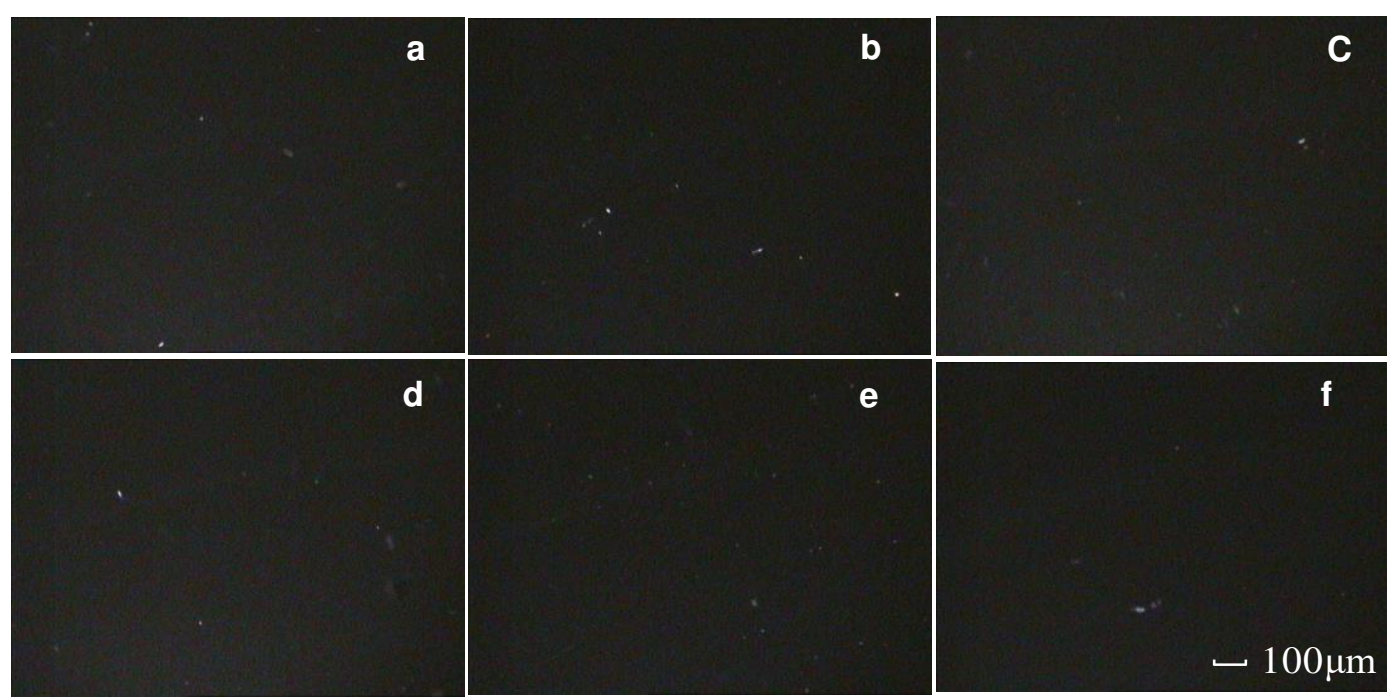

Fig.5 Polarized optical microscopy images of the ND MCC dissolved in the different $\mathrm{NaOH} /$ additives aqueous solutions with the direct dissolution method: (a) $7 \% \mathrm{NaOH} / 12 \%$ urea, (b) $9.5 \% \mathrm{NaOH} / 4.5 \%$ thiourea, and (c) $8 \% \mathrm{NaOH} / 8 \%$ urea/6.5\% thiourea; and with the freezing-thaw method: (d) $7 \% \mathrm{NaOH} / 12 \%$ urea, (e)

$9.5 \% \mathrm{NaOH} / 4.5 \%$ thiourea, and (f) $8 \% \mathrm{NaOH} / 8 \%$ urea/6.5\% thiourea

361 Fig.4 and Fig.5 illustrates the dissolving behavior of the MCC samples in different $362 \mathrm{NaOH} /$ additives aqueous solutions using the direct dissolution and freezing-thaw methods. OD 
363 MCC yielded better dissolution using the direct dissolution method than using the freezing-thaw 364 method. It seems that ND MCC yielded similar dissolution using two dissolution methods, which 365 indicated that the direct dissolution method was best suited for cellulose with a lower DP. And 366 there was no discernible difference in the effects of the two dissolution methods on the dissolution of $\mathrm{MCC}$ in the $8 \% \mathrm{NaOH} / 8 \%$ urea/6.5\% thiourea solution.

\section{Conclusions}

370 The dissolving capacity of the $\mathrm{NaOH} /$ additives aqueous solutions was dependent on the origin 371 of the pulp fibers, composition of the aqueous solution, and dissolving method. The softwood pulp 372 had a better dissolution when it was prepared in the ND state rather than the OD state. Dryness had 373 no remarkable effect on the hardwood pulp. The direct dissolution method was best suited for OD 374 cellulose fibers with a low DP and the freezing-thaw method was best suited for ND cellulose 375 fibers with a high DP. The dissolving method used had a big difference in the solubility for the 7\% $376 \mathrm{NaOH} / 12 \%$ urea and $9.5 \% \mathrm{NaOH} / 4.5 \%$ thiourea solutions. However, the dissolving method used 377 had little difference in the solubility for the $8 \% \mathrm{NaOH} / 8 \%$ urea/6.5\% thiourea solution. The $8 \%$ $378 \mathrm{NaOH} / 8 \%$ urea/6.5\% thiourea solution had the best dissolution capabilities for all of the pulp 379 samples and both dissolving methods. The SDP was able to achieve an $80 \%$ dissolved proportion 380 in the $8 \% \mathrm{NaOH} / 8 \%$ urea/6.5\% thiourea solution.

Acknowledgments The authors would like to acknowledge Hongjie Zhang for DP of SDP and contents of the neutral sugars of the three pulps.

Author Contributions WK: Dissolution experiment, Investigation of polarized optical microscopy, Writing-Original Draft, Plotting. GY: testing of $S_{10}$ value, $S_{18}$ value, and intrinsic viscosity, Investigation. JX: fiber parameters of the three pulps. ML: Conceptualization, Resources, Writing-Review and Editing. YS: Conceptualization, Resources, Review and Editing, Project administration, Funding acquisition, Supervision.

Funding This study was supported by the National Natural Science Foundation of China (no.51403156), by and by the National Innovation Training Program for College Students (no. 201810069029).

Data Availability Not applicable. Code Availability Not applicable.

Consent for Publication All authors consent for publication in Journal of Polymers and the Environment.

\section{Compliance with Ethical Standards}

Conflict of interest The authors declared that they have no conflicts of interest to this work. We declare that we do not have any commercial or associative interest that represents a conflict of interest in connection with

402 Ethical approval Not applicable.

403 Informed Consent Not applicable.

\section{References}

406 1. Cai J, Zhang L (2005) Rapid dissolution of cellulose in LiOH/urea and NaOH/urea aqueous solutions. 
2. Isogai A, Atalla RH (1998) Dissolution of cellulose in aqueous NaOH solutions. Cellulose 5(4), 309-319. https://doi.org/10.1023/a:1009272632367

3. Gavillon R, Budtova T (2007) Kinetics of cellulose regeneration from cellulose-NaOH-water gels and comparison with cellulose-N-methylmorpholine-N-oxide-water solutions. Biomacromolecules 8(2), 424 432. https://doi.org/10.1021/bm060376q

4. Qi H, Chang C, Zhang L (2008) Effects of temperature and molecular weight on dissolution of cellulose in $\mathrm{NaOH} / \mathrm{urea}$ aqueous solution. Cellulose 15(6), 779-787. https://doi.org/10.1007/s10570-008-9230-8

5. Lue A, Zhang L, Ruan D (2007) Inclusion complex formation of cellulose in $\mathrm{NaOH}$-thiourea aqueous system at low temperature.Macromol Chem Phys 208(21), 2359-2366. https://doi.org/10.1002/macp.200700177

6. Luo X, Zhang L (2013) New solvents and functional materials prepared from cellulose solutions in alkali/urea aqueous system. Food Res Int 52(1), 387-400. https://doi.org/10.1016/j.foodres.2010.05.016

7. Zhang L, Ruan D, Gao S (2002) Dissolution and regeneration of cellulose in $\mathrm{NaOH} /$ thiourea aqueous solution. J Polym Sci Pol Phys 40(14), 1521-1529. https://doi.org/10.1002/polb.10215

8. Jin H, Zha C, Gu L (2007) Direct dissolution of cellulose in $\mathrm{NaOH} /$ thiourea/urea aqueous solution. Carbohyd Res 342(6), 851-858. https://doi.org/10.1016/j.carres.2006.12.023

9. Zhang S, Li F-X, Yu J-y et al (2010) Dissolution behavior and solubility of cellulose in NaOH complex solution. Carbohyd Polym 81(3), 668-674. https://doi.org/10.1016/j.carbpol.2010.03.029

10. Qin X, Lu A, Cai J et al (2013) Stability of inclusion complex formed by cellulose in $\mathrm{NaOH} /$ urea aqueous solution at low temperature. Carbohyd Polym 92(2), 1315-1320. https://doi.org/10.1016/j.carbpol.2012.10.004

11. Yang Q, Qi H, Lue A et al (2011) Role of sodium zincate on cellulose dissolution in $\mathrm{NaOH} /$ urea aqueous solution at low temperature. Carbohyd Polym 83(3), 1185-1191. https://doi.org/10.1016/j.carbpol.2010.09.020

12. Zhang S, Li F, Yu J (2009) Preparation of cellulose/chitin blend bio-fibers via direct dissolution.Cellulose Chem Technol 43:393-398

13. Kihlman M, Medronho BF, Romano AL et al (2013) Cellulose dissolution in an alkali based solvent: influence of additives and pretreatments. J Braz Chem Soc 24:295-303. https://doi.org/10.5935/01035053.20130038

14. Zhou J, Zhang L (2000) Solubility of cellulose in NaOH/urea aqueous solution. Polym J 32, 866-870. https://doi.org/ 10.1295/polymj.32.866

15. Yan L, Gao Z (2008) Dissolving of cellulose in PEG/NaOH aqueous solution.Cellulose 15, 789-796. https://doi.org/10.1007/s10570-008-9233-5

16. Zhao DS, Li H, Liu MS et al (2011) Dissolubility of the cellulose in urea/caprolactam/ $\mathrm{NaOH} /$ aqueous solution and the performance of regenerated cellulose. Chem J Chinese U 32(7), 1629-1633

17. Shi Y, Zhao SS, Ji NN et al (2016) Quality analysis of dissolving pulp cellulose fibers in NaOH/additives aqueous solution. Polym Bull 12, 43-49

18. Shi Y, Zhang K, Sun H et al (2017) Dissolution behavior of higher DP bamboo dissolving pulp fiber in $\mathrm{NaOH} /$ additive aqueous solution. Journal of Functional Materials 48(6), 6001-6006. https://doi.org/10.3969/j.issn.1001-9731.2017.06.014

19. Shi Y, Kong R, Yang F et al (2018) Participation of sodium sulfamate as hydrogen bond-donating and hydrogen bond-accepting additive in the dissolution of cellulose in $\mathrm{NaOH}$ aqueous solution. Cellulose 25(5), 2785-2794. https://doi.org/ 10.1007/s10570-018-1731-5

20. Brännvall E (2007) Aspects on Strength Delivery and Higher Utilisation of the Strength Potential of Softwood Kraft Pulp Fibres, Ph.D. Dissertation, KTH Royal Institute of Technology, Stockholm, 
454 21. Gerber PJ, Heitmann JA, Joyce TW et al (1999) Adsorption of hemicellulases onto bleached kraft fibers. J Biotechnol 67(1), 67-75. https://doi.org/10.1016/s0168-1656(98)00163-1

456 22. Ibbett RN, Kaenthong S, Phillips DAS et al (2007) Solute adsorption and exclusion studies of the structure of never-dried and re-wetted cellulosic fibres. J Mater Sci 42(16), 6809-6818. https://doi.org/ 10.1007/s10853-006-1426-4

459 23. Spinu M, Dos Santos N, Le Moigne N et al (2011) How does the never-dried state influence the swelling and dissolution of cellulose fibres in aqueous solvent?.Cellulose 18(2) 247-256. https://doi.org/ 10.1007/s10570-010-9485-8

462 24. Egal M, Budtova T, Navard P (2008) The dissolution of microcrystalline cellulose in sodium hydroxide463 urea aqueous solutions. Cellulose 15(3), 361-370. https://doi.org/10.1007/s10570-007-9185-1 


\section{Figures}
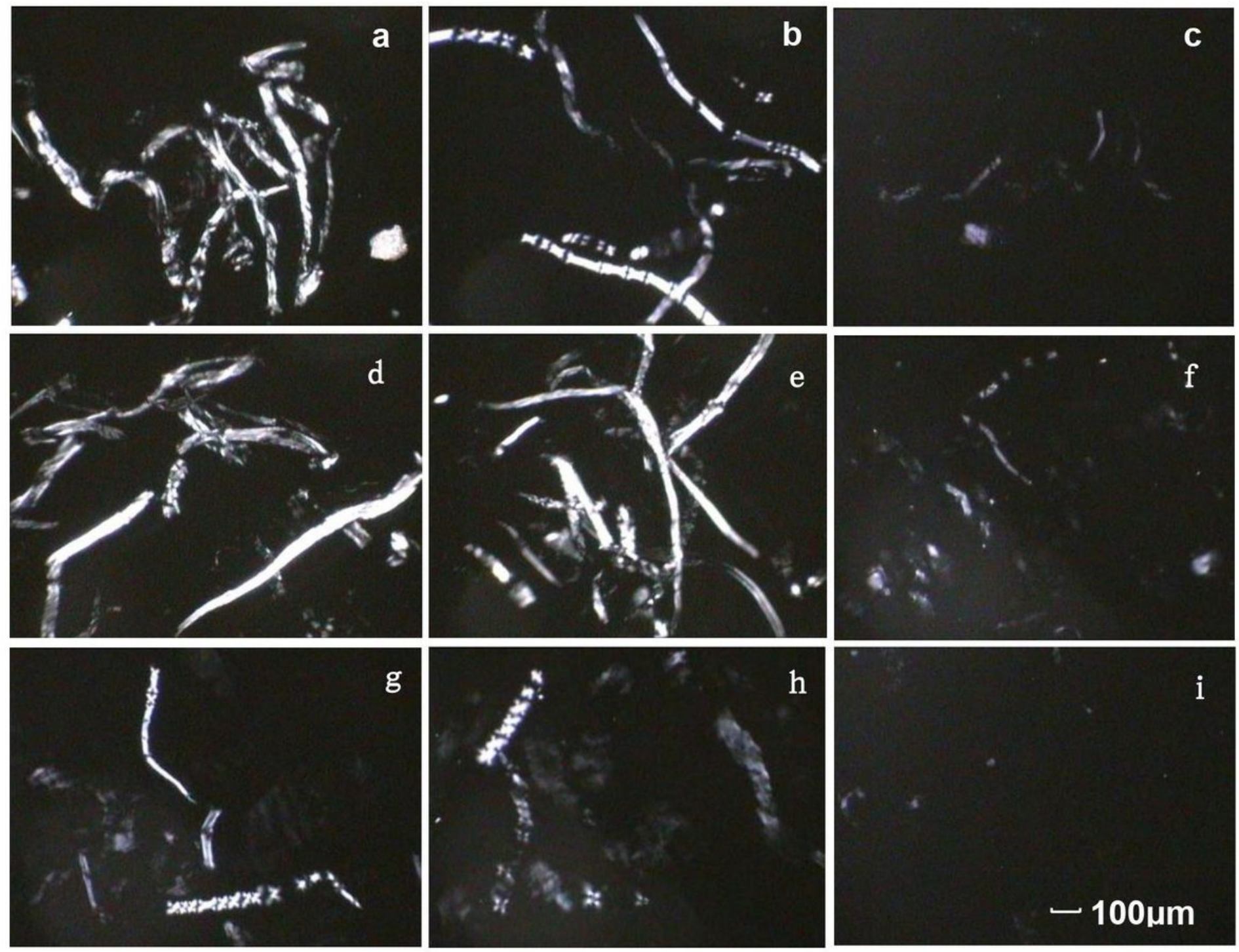

\section{Figure 1}

Polarized optical microscopy images of the SDP dissolved in the different $\mathrm{NaOH}$ /additives aqueous solutions using direct dissolution method,OD: (a) 7\% NaOH/12\% urea, (b) $9.5 \% \mathrm{NaOH} / 4.5 \%$ thiourea, and (c) $8 \% \mathrm{NaOH} / 8 \%$ urea/6.5\% thiourea; using direct dissolution method,ND: (d) $7 \% \mathrm{NaOH} / 12 \%$ urea, (e) 9.5\% NaOH/4.5\% thiourea, and (f) $8 \% \mathrm{NaOH} / 8 \%$ urea/6.5\% thiourea and using freezing-thaw method,ND: (g) $7 \% \mathrm{NaOH} / 12 \%$ urea, (h) $9.5 \% \mathrm{NaOH} / 4.5 \%$ thiourea, and (i) $8 \% \mathrm{NaOH} / 8 \%$ urea/6.5\% thiourea 

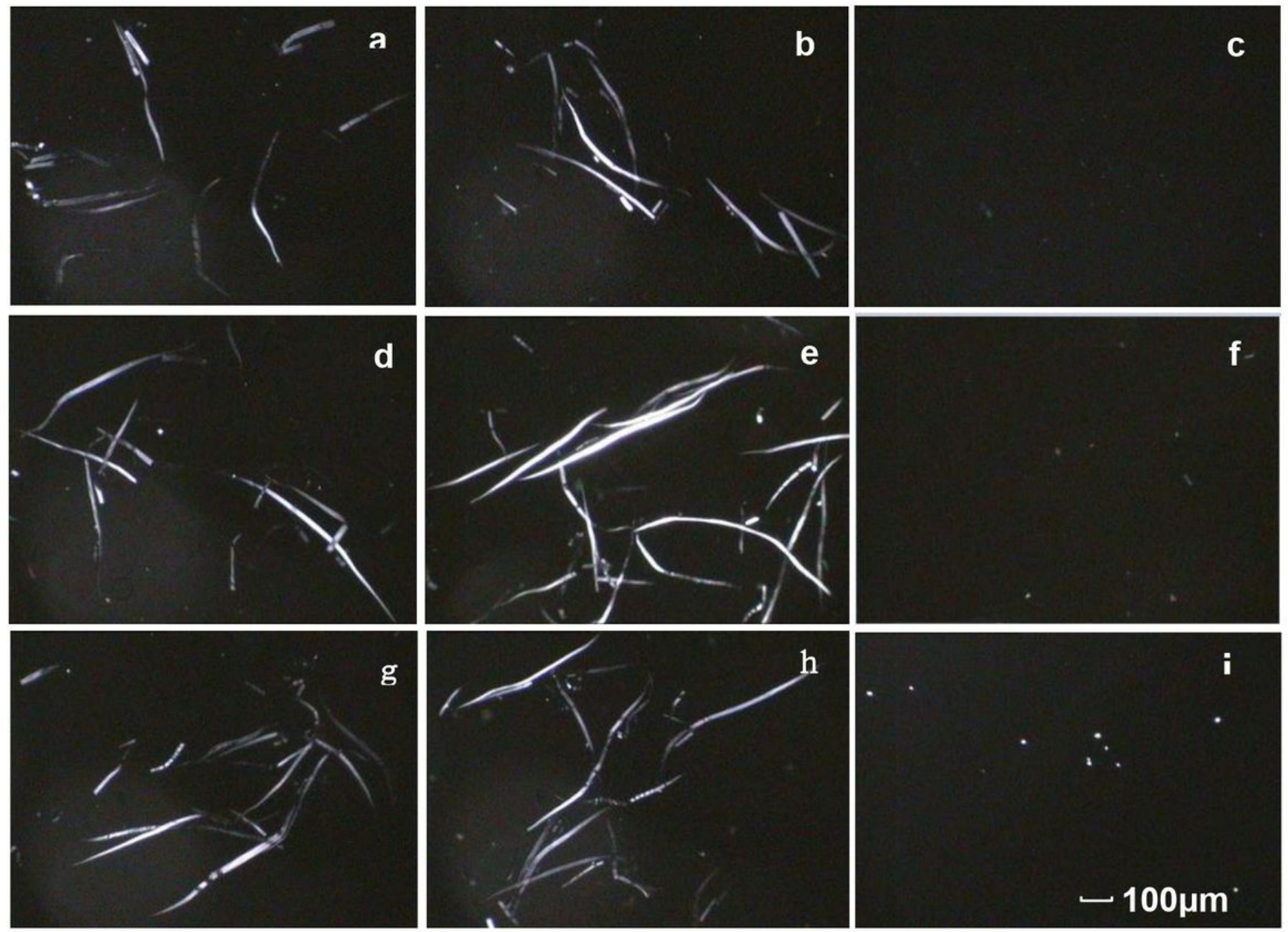

\section{Figure 2}

Polarized optical microscopy images of the HDP dissolved in the different $\mathrm{NaOH}$ /additives aqueous solutions using direct dissolution method,OD: (a) 7\% NaOH/12\% urea, (b) $9.5 \% \mathrm{NaOH} / 4.5 \%$ thiourea, and (c) $8 \% \mathrm{NaOH} / 8 \%$ urea/6.5\% thiourea; using direct dissolution method,ND: (d) $7 \% \mathrm{NaOH} / 12 \%$ urea, (e) 9.5\% NaOH/4.5\% thiourea, and (f) $8 \% \mathrm{NaOH} / 8 \%$ urea/6.5\% thiourea and using freezing-thaw method,ND: (g) $7 \% \mathrm{NaOH} / 12 \%$ urea, (h) $9.5 \% \mathrm{NaOH} / 4.5 \%$ thiourea, and (i) $8 \% \mathrm{NaOH} / 8 \%$ urea/6.5\% thiourea 

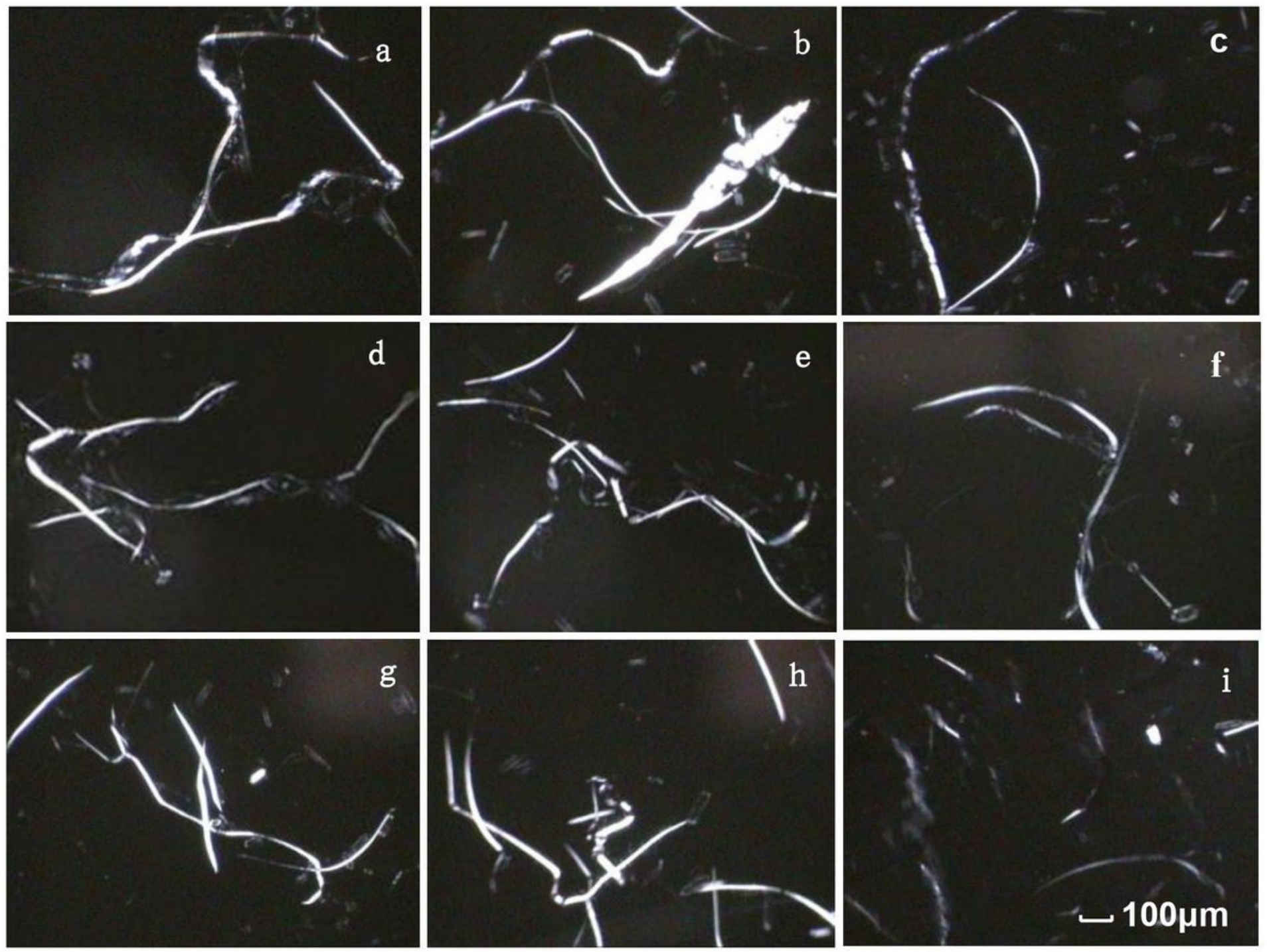

\section{Figure 3}

Polarized optical microscopy images of the BDP dissolved in the different $\mathrm{NaOH}$ /additives aqueous solutions using direct dissolution method,OD: (a) 7\% NaOH/12\% urea, (b) $9.5 \% \mathrm{NaOH} / 4.5 \%$ thiourea, and (c) $8 \% \mathrm{NaOH} / 8 \%$ urea/6.5\% thiourea; using direct dissolution method,ND: (d) $7 \% \mathrm{NaOH} / 12 \%$ urea, (e) 9.5\% NaOH/4.5\% thiourea, and (f) $8 \% \mathrm{NaOH} / 8 \%$ urea/6.5\% thiourea and using freezing-thaw method,ND: (g) $7 \% \mathrm{NaOH} / 12 \%$ urea, (h) $9.5 \% \mathrm{NaOH} / 4.5 \%$ thiourea, and (i) $8 \% \mathrm{NaOH} / 8 \%$ urea/6.5\% thiourea 


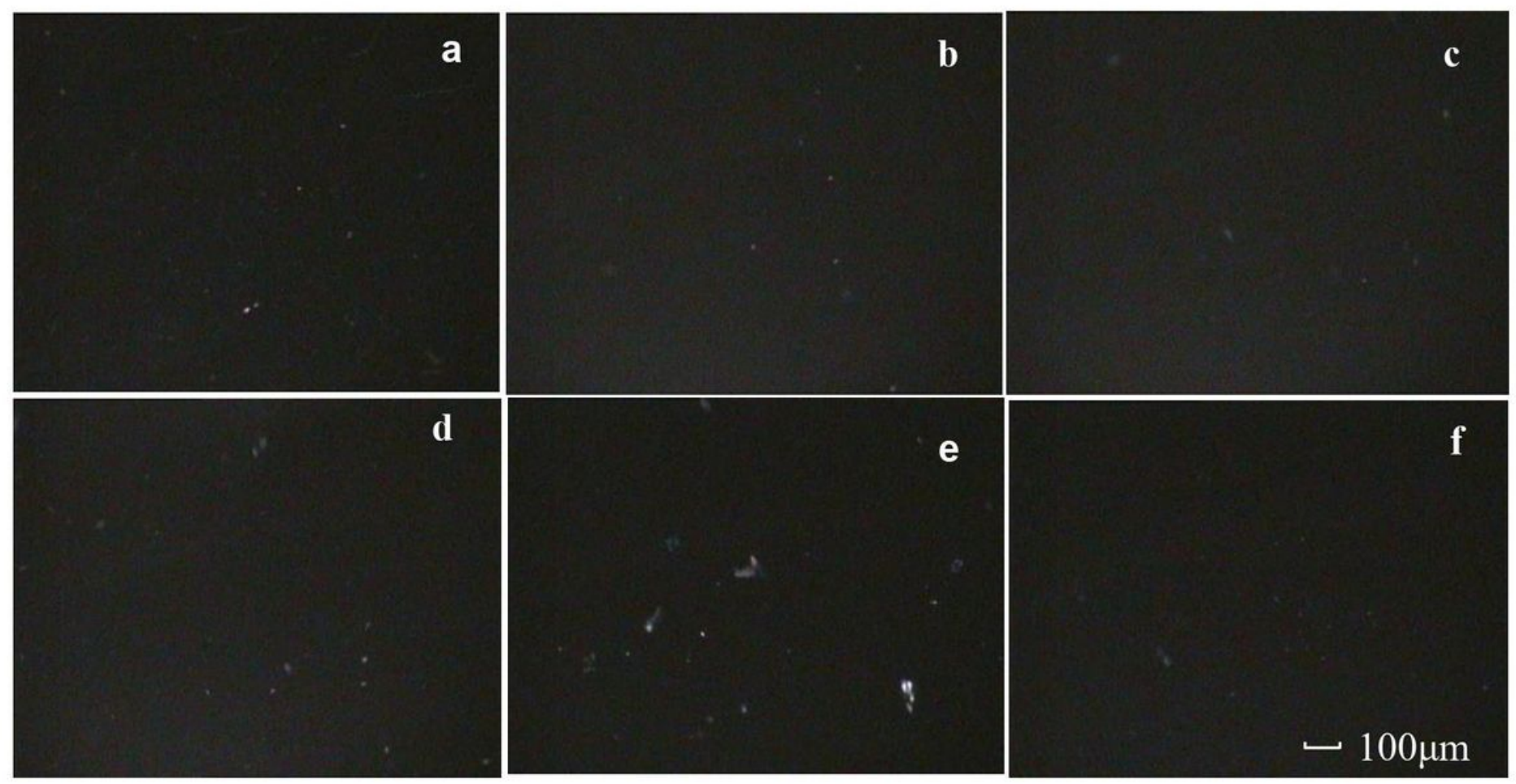

\section{Figure 4}

Polarized optical microscopy images of the OD MCC dissolved in the different $\mathrm{NaOH}$ /additives aqueous solutions with the direct dissolution method: (a) 7\% NaOH/12\% urea, (b) $9.5 \% \mathrm{NaOH} / 4.5 \%$ thiourea, and (c) $8 \% \mathrm{NaOH} / 8 \%$ urea/6.5\% thiourea; and with the freezing-thaw method: (d) $7 \% \mathrm{NaOH} / 12 \%$ urea, (e) $9.5 \% \mathrm{NaOH} / 4.5 \%$ thiourea, and (f) $8 \% \mathrm{NaOH} / 8 \%$ urea/ $6.5 \%$ thiourea

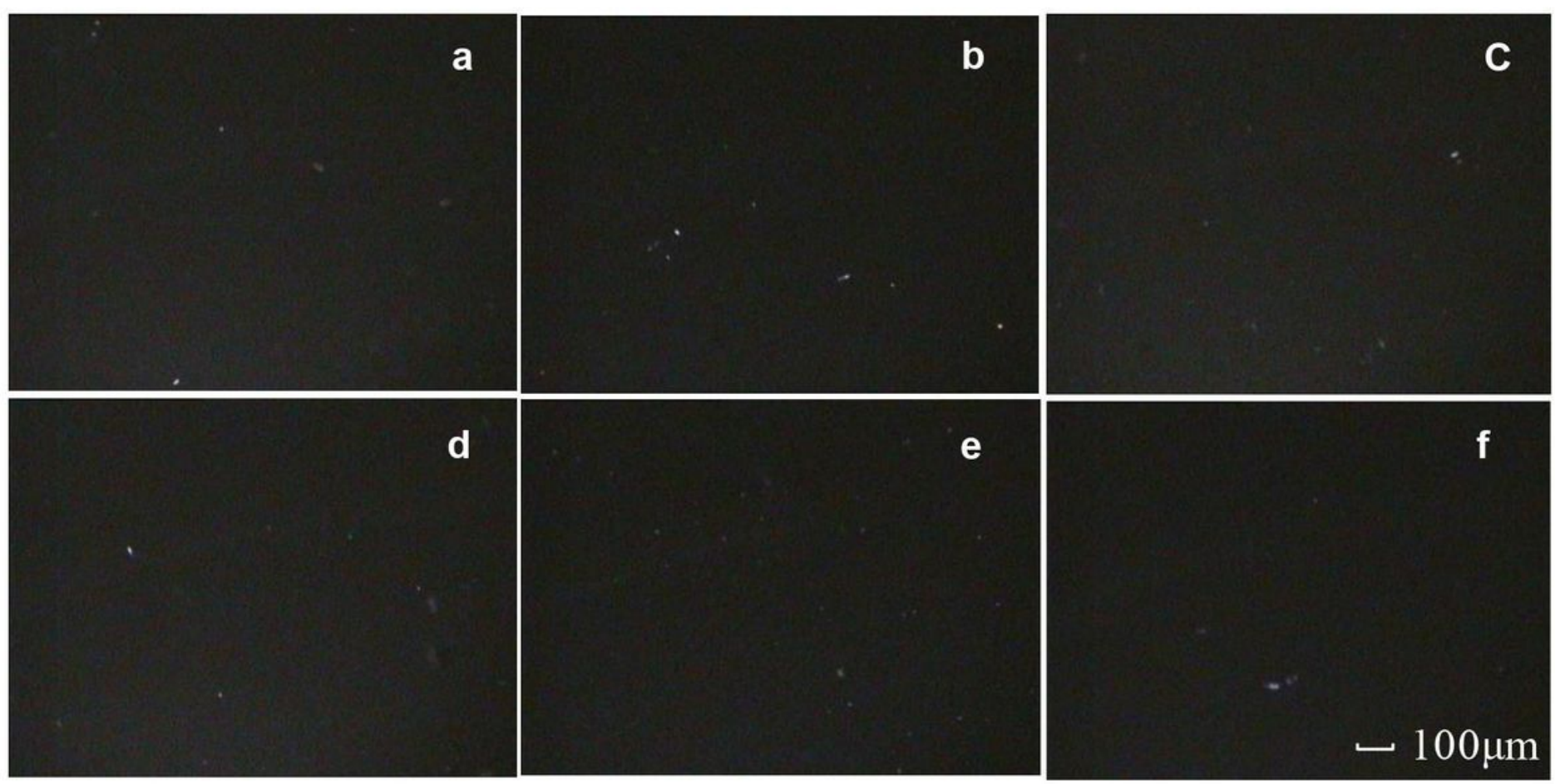




\section{Figure 5}

Polarized optical microscopy images of the ND MCC dissolved in the different $\mathrm{NaOH}$ /additives aqueous solutions with the direct dissolution method: (a) 7\% NaOH/12\% urea, (b) $9.5 \% \mathrm{NaOH} / 4.5 \%$ thiourea, and (c) $8 \% \mathrm{NaOH} / 8 \%$ urea/6.5\% thiourea; and with the freezing-thaw method: (d) $7 \% \mathrm{NaOH} / 12 \%$ urea, (e) $9.5 \% \mathrm{NaOH} / 4.5 \%$ thiourea, and (f) $8 \% \mathrm{NaOH} / 8 \%$ urea/ $6.5 \%$ thiourea 